\title{
Seminare / Séminaires 2010
}

\section{Praxiscomputerworkshop}

\section{Inhalt}

Die Workshopteilnehmer/innen erhalten im 1. Teil eine Einführung in die Anforderungen an ein Praxisinformationssystem. Anhand einer modernen vernetzten Praxisinfrastruktur werden die Beurteilungskriterien für eine praxis- und zukunftstaugliche Softwarelösung dargestellt. Checklisten sollen die schnelle Orientierung unterstützen und bei der Beurteilung und Wahl des Produkts konkrete Hilfe bieten. In Zusammenarbeit mit SGAM.Informatics werden die zentralen Elemente der elektronischen Krankengeschichte aufgezeigt. Ein Erfahrungsbericht eines EDV-Anwenders (Arzt) rundet den 1. Teil ab. Der 2. Teil umfasst die Präsentation von sechs Praxisadministrationssoftwarelösungen (Leistungserfassung, elektronisches Abrechnen unter Einbezug der TrustCenter, Agendaführung, Statistiken, Laborgeräteeinbindung, elektronische Krankengeschichte, Finanzbuchhaltungslösungen usw.).

\section{Ziel}

Die Teilnehmer/innen erhalten einen Anforderungskatalog, welcher ihnen erlaubt, ihre Vorstellungen für ein modernes Praxisinformationssystem besser $\mathrm{zu}$ formulieren und diese dem Softwarehersteller zu dessen Vorbereitung zu kommunizieren. Zudem erhalten sie einen ersten Überblick über führende Softwarelösungen.

\section{Daten}

K15 Donnerstag, 25. November 2010 Stadttheater 9.30-16.30 Uhr Olten

\section{Folgende Softwareanbieter können Sie} im 2. Teil des Workshops kennenlernen:

\section{Ärztekasse, Urdorf (CB 7)}

Als Marktleader seit 1964 im Bereich Praxisadministration können Sie bei der Ärztekasse auf eine ganzheitliche Lösung für Ihre Fragen betreffend Abrechnungsvarianten, Computerwahl, Netzwerke, Formular- und Briefbearbeitung usw. zählen. Die statistischen Erhebungen (im Auftrag der FMH Roko) sind ein anerkanntes betriebswirtschaftliches und standesorganisatorisches Hilfsmittel für die moderne Praxisführung. Nebst innovativen Ideen und Lösungen sowie Kooperationspartnerschaft mit 10 TrustCenter ist uns eine umfassende Kundenbetreuung ein Anliegen. Unsere Standorte befinden sich in Basel, Bern, Chur, Crissier, Genf, Lugano, Luzern, Neuchâtel, St. Gallen, Thônex und Zürich. Sie definieren Ihre Wünsche wir bieten Ihnen die Lösung!
Delemed AG Medical Software, Kehrsatz (PEX II) Delemed AG entwickelt bereits über 18 Jahre erfolgreich Medizinsoftware für die Praxen. Die Software besticht durch den sympathischen, effizienten, einfachen und modularen Aufbau und lässt in keiner Praxis Wünsche offen. Dank unserer Vielseitigkeit im medizinischen Umfeld sind wir Ihr optimaler Partner.

\section{Gartenmann Software AG,} Seuzach (PRAXIS*DESKTOP)

Kompetent, effizient und innovativ

An diesen Werten orientieren wir uns seit der Gründung der Gartenmann Software AG 1992. Den Ausgangspunkt all unserer Überlegungen und Tätigkeiten bilden dabei immer unsere Kunden. PRAXIS*DESKTOP ist mit den neusten Technologien ausgestattet und überzeugt durch eine intuitive und effiziente Benutzerführung. Durch die nahe Zusammenarbeit mit unseren Partnern sind wir in der Lage, Praxis-Desktop rasch und effizient weiterzuentwickeln.

\section{Kern Concept AG, Gossau SG (AESKULAP)}

AESKULAP ist ein extrem effizientes Administrationsprogramm, das zusammen mit einer SQL-Datenbank höchste Verarbeitungsgeschwindigkeit, Kompaktheit und schnelle Zugriffszeiten gewährleistet. AESKULAP bietet für jedes Budget und für alle Bedürfnisse eine optimale und intelligente Lösung: Vom einfachen Abrechnungssystem mit sämtlichen Leistungserfassungsmöglichkeiten bis hin zur führenden vollstrukturierten elektronischen KG-Führung, die in der Praxis keine Wünsche offenlässt. AESKULAP ist ein modular aufgebautes und anpassbares System mit einer Vielzahl von Modulen. Unsere extrem preisgünstige Basisversion kann somit bei Bedarf jederzeit erweitert werden. AESKULAP, die intelligente Ärztesoftware für jedes Budget!

\section{HCI Solutions AG, Gümligen (TriaMed ${ }^{\circledR}$ )}

Die Abteilung Triamun von HCI Solutions, ein Unternehmen der Galenica-Gruppe, entwickelt und vermarktet innovative Softwarelösungen für das Praxis-, Apotheken- und Unternehmensmanagement. Individuelle Beratung, die gesamte Soft- und Hardware, einen umfassenden Support und Schulung aus einer Hand. Unsere Softwarelösung TriaMed ${ }^{\circledR}$ für Arztpraxen, Gruppenpraxen, medizinische Zentren und Ärztenetzwerke basiert auf der neusten Technologie und wurde zusammen mit Ärzten entwickelt. So ist eine intuitiv bedienbare Praxismanagement-Lösung entstanden, die sämtliche Bedürfnisse von integriertem und vernetztem Arbeiten befriedigen.

Um den stetig wachsenden Anforderungen an eine praxisgerechte Softwarelösung gerecht $\mathrm{zu}$ werden, wird TriaMed $^{\circledR}$ stetig weiterentwickelt. Folgende 
wichtige Neuerungen wurden im vergangenen Jahr vorgenommen: Sichtenkonzept zur individuellen arztspezifischen Gestaltung der Krankengeschichte, Versandmappe, Medizinisches Auswertungstool, Elektronisches Rezept, Versicherungscheck, PatientenBonitätsprüfung, Schnellarchivierung, Mail-Attachements per Drag\&Drop.

\section{TMR - Triangle Micro Research AG,} Hölstein (WinMed ${ }^{\circledR}$ )

Die TMR AG wurde von Ärzten, Wissenschaftlern, Unternehmern und Dozenten der Uni Basel 1993 als «Spin-Off»-Firma gegründet. Den Schwerpunkt in der Entwicklung der TMR AG bilden Anwendungen im Bereich der med. Telekommunikation, Internet sowie der mehrmandanten- und mehrplatzfähigen Ärztesoftware WinMed ${ }^{\circledast}$. WinMed ${ }^{\circledR}$ ist ein vollständiges, äusserst einfach zu bedienendes Arztpraxisadministrationssystem mit integrierter Kommunikationsplattform, Bild- und Dokumentenverwaltung sowie modernster elektronischer Krankengeschichte. WinMed ${ }^{\circledR}$ wird nach ausgereifter mehrjähriger Pilotphase erfolgreich seit 1998 verkauft und zählt heute zu den meistgekauften Ärztesoftwarepaketen. Vertrieben wird WinMed ${ }^{\circledR}$ in der Deutschschweiz von TMR AG selbst und im Tessin von GFP Mediconsul in Massagno.

\section{Praxisübergabe}

Das Seminar richtet sich an zukünftige Praxisübergeber/innen. Hinweis: Vor allem aus steuerplanerischer Sicht lohnt es sich, sich bereits frühzeitig (510 Jahre) mit diesem Thema auseinanderzusetzen.

\section{Themen}

Juristische Aspekte (Praxisübergabevertrag, allg. Vertragswesen, Übergabe der Krankengeschichten), Unternehmensbewertung einer Arztpraxis (Berechnung Inventarwert und Goodwill als Verhandlungsbasis), Versicherungen/Vorsorge/Vermögen (Übergabe/ Auflösung von Versicherungsverträgen, Pensions- und Finanzplanung), Steuern (Steueraspekte bei der Praxisübergabe, Optimierung der steuerlichen Auswirkungen, Liquidations- und Grundstückgewinnsteuer, Bestimmung des optimalen Übergabezeitpunktes).

\section{Sponsoren}

Die Kosten werden durch diverse Sponsoren (siehe www.fmhservices.ch) gedeckt.

\section{Daten}

K10 Donnerstag, 11. November 2010 Hotel Victoria 13.30-18.00 Uhr Basel

\section{Ouverture et reprise d'un cabinet médical}

\section{Contenu}

Business plan (préparation du plan de financement et crédit d'exploitation, financement par la banque), Aménagement (implantation, projet et concept d'aménagement, choix du mobilier, budget), Estimation d'un cabinet (inventaire et goodwill), Administration d'un cabinet médical (dans le cabinet, par la banque), Assurances (toutes les assurances à l'intérieur et autour du cabinet), Passage du statut de salarié à celui d'indépendant et fiscalité.

\section{Sponsors}

Les coûts sont pris en charge par divers sponsors (voir www.fmhservices.ch).

\section{Dates}

$\begin{array}{lll}\text { K23 Jeudi, } 18 \text { novembre } 2010 & \text { World Trade } \\ 17.00-21.30 \mathrm{~h} & \text { Center Lausanne }\end{array}$

\section{Remise d'un cabinet médical}

\section{Contenu}

Aspects juridiques (autour du contrat de remise/ reprise), Estimation d'inventaire et goodwill d'un cabinet, Assurances (prévoyance, assurances à l'intérieur et autour du cabinet), Conséquences fiscales d'une remise.

\section{Sponsors}

Les coûts sont pris en charge par divers sponsors (voir www.fmhservices.ch).

\section{Dates \\ K25 Jeudi, 11 novembre 2010 \\ Hôtel du Parc $17.00-21.30 \mathrm{~h}$ des Eaux-Vives Genève}

\section{Anmeldung und Auskunft / Inscription et information}

www.fmhservices.ch oder FMH Consulting Services, Cornelia Steinmann, Burghöhe 1, 6208 Oberkirch, Tel. 04192500 77, Fax 0419210586

\section{Hinweis / Remarque}

Bei sämtlichen Seminaren, bei denen die Kosten teilweise oder gänzlich von Seminarsponsoren gedeckt werden, werden die Teilnehmeradressen den jeweiligen Sponsoren zur Verfügung gestellt.

Les adresses des participants aux séminaires dont les coûts sont couverts en partie ou totalement par des sponsors sont communiquées aux sponsors concernés.

\section{Annullierungsbedingungen /}

Conditions d'annulation

Bei Abmeldungen oder Fernbleiben werden folgende Unkostenbeiträge erhoben:

Un montant est perçu pour une absence ou une annulation. Il est de:

- 50 CHF pro Person ab 14 Tage vor Seminarbeginn / par personne dans les 15 jours avant le début du séminaire;

- 100 CHF pro Person ab 7 Tage vor Seminarbeginn oder Fernbleiben / par personne dans les 7 jours avant le début du séminaire. 


\section{Seminarsponsoren 2010}

Die Unterstützung durch verschiedene Sponsoren ermöglicht es der FMH Consulting Services AG, ihre Seminarreihen für FMH-Mitglieder teils kostenlos, teils kostengünstig anzubieten. Gerne stellen wir Ihnen diese Firmen in einem Kurzporträt vor.

\section{medics labor}

professionell und persönlich

Medics Labor AG

Chutzenstrasse 24, 3001 Bern

Tel. 03137220 02, Fax 0313714044

info@medics-labor.ch

www.medics-labor.ch

\section{Medizinisches Labor und mehr}

Medics Labor ist ein Schweizer Unternehmen, zu Hause in Bern, hier verwurzelt und seit vielen Jahren erfolgreich tätig im Kanton sowie weiteren Regionen. Geschätzt als persönliches, unkompliziertes Gegenüber, überzeugt Medics Labor durch fachliches und menschliches Gespür mit zahlreichen Hilfestellungen und Dienstleistungen. Wir verstehen uns als sozialer Arbeitgeber und beschäftigen auch behinderte Personen. Medics Labor ist ein Labor von Ärzten für Ärzte. Es gehört den Laborspezialisten und den Ärzten, die das Unternehmen gemeinsam führen.

\section{BIOEANALYTICA \\ Medizinisches Labor}

Bio-Analytica AG

Maihofstrasse 95a, 6006 Luzern

Tel. 04142931 31, Fax 0414293130

service@bioanalytica.ch

www.bioanalytica.ch

\section{Engagierte Kompetenz}

Bio-Analytica, 1957 in Luzern gegründet, basiert auf einer langjährigen Tradition. Stetige Innovation und ein Team qualifizierter Fachspezialisten und Labormediziner bilden das Fundament unserer Kompetenz. Qualität und Seriosität - das sind die Werte, denen wir uns verschrieben haben. Aus der Überzeugung, dass dies auch unseren Kunden wesentliche Vorteile bietet, haben wir unser Labor im Jahre 2000 akkreditieren lassen.

Schnell, wenn notwendig auch rund um die Uhr, sind wir für Sie da. Mit dem Know-how von über 80 Mitarbeitenden, modernster Laborautomation und Informationstechnologie sind unsere Laborresultate in kürzester Zeit verfügbar.

Bei Bio-Analytica stehen Sie als Kunde im Mittelpunkt. Wir unterstützen Sie und Ihr Praxisteam jederzeit gerne optimal im persönlichen Kontakt und mit zahlreichen wertvollen zusätzlichen Dienstleistungen.

\section{Laborgemeinschaft \\ Medizinisches Labor \\ Laborgemeinschaft 1 \\ Rautistrasse 11, 8047 Zürich \\ Tel. 04440420 80, Fax 0444042088 \\ lg1@lg1.ch \\ www.lg1.ch}

\section{Praxisnähe und Eigenständigkeit}

Die Laborgemeinschaft 1, gegründet 1979 als genossenschaftlich geführtes medizinisches Labor, hat bis heute ihre Selbständigkeit bewahrt. Werte wie finanzielle Unabhängigkeit, individuelle Kundenbetreuung, Engagement in der Lehrlingsausbildung und der Bezug zur Region stehen bei uns im Vordergrund.

Darauf können Sie zählen: 40 motivierte Mitarbeiterinnen und Mitarbeiter; Support bei labordiagnostischen Fragen durch ein routiniertes und medizinisch ausgebildetes Fachteam; modernste Analysengeräte; Kommunikation mit fortschrittlichster EDV-Technologie; spezifische Unterstützung durch unseren Kundendienst. Weitere Dienstleistungen erfahren Sie auf: www.lg1.ch oder www.mkz.ch

Für Sie und Ihr Personal bieten wir zudem in unserem Kurszentrum Fortbildungen an, weil uns Ihre und unsere Weiterbildung wichtig ist. Auch darin sehen wir einen wichtigen Beitrag für eine seriöse, patientenorientierte und kostengünstige Diagnostik.

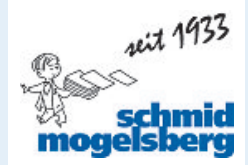

P. Schmid + Co. AG, Ärztedrucksachen Sonnmattstrasse 1, 9122 Mogelsberg Tel. 07137560 80, Fax 0713756081 info@schmid-mogelsberg.ch www.schmid-mogelsberg.ch

Seit über 70 Jahren auf Ärztedrucksachen spezialisiert!

Bei der Gestaltung von zweckmässigen Arztformularen (Patientenkarten, KG-Einlagenblätter usw.) profitieren Sie von der langjährigen Erfahrung. Die Vergangenheit hat gezeigt, dass sich kaum zwei Ärzte für den gleichen Druck entscheiden. Zweckmässige Materialauswahl, einwandfreie Verarbeitung, freundliche und kompetente Beratung, schnelle Lieferung und die Ausführung von Spezialwünschen - diese Dienstleistungen schaffen die Grundlage für ein langjähriges Vertrauensverhältnis. Auf Wunsch versenden wir eine individuell auf Ihre Fachrichtung zusammengestellte Druckmusterkollektion.

An über 9000 Ärzte liefern wir Drucksachen, Papiere und Büroartikel für den Praxisalltag. 


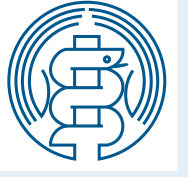

Schweizerische Ärzte-Krankenkasse

Oberer Graben 37, 9001 St. Gallen

Tel. 07122718 18, Fax 0712271828

info@saekk.ch

www.saekk.ch

Die richtige Adresse für Erwerbsausfalldeckungen, Kollektivkrankenkasse und Versicherungsplanung Mit mehr als 100 Jahren Erfahrung kennt unsere Organisation auch heute die Bedürfnisse der Ärztinnen und Ärzte. Sie bietet entsprechend durchdachte und kostengünstige Lösungen an, sowohl für Praxiseröffner/innen wie auch für selbständige und angestellte Ärztinnen und Ärzte.

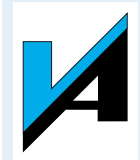

Versicherung der Schweizer Ärzte Genossenschaft Länggassstrasse 8, 3000 Bern 9

Tel. 03130125 55, Fax 0313025156

versa@versa.ch

www.versa.ch

Spezialisiert auf die Bedürfnisse von Ärztinnen und Ärzten und deren Ehegatten, bietet die Versicherung der Schweizer Ärzte Genossenschaft individuelle, den jeweiligen Bedürfnissen angepasste Versicherungslösungen im Bereich der privaten Vorsorge an.

\section{Unilabs}

www.unilabs.ch

\section{UNILABS - Ihr Qualitätslabor}

schnell, zuverlässig und nah

Unilabs ist im Bereich der medizinischen Analysen ein kompetenter, transparenter und zuverlässiger Partner. Wir bieten Ihnen überall in der Schweiz ein komplettes Analysenspektrum, umfassende Dienstleistungen und kompetente Fachberatung an. In der Deutschschweiz sind dies Unilabs Mittelland mit den Standorten Basel, Bern, Burgdorf, Langnau, Solothurn, Thun; Unilabs Zürich und Unilabs Dr. Weber. Unilabs bietet nicht nur medizinische Laboranalysen, sondern auch wertvolle individuelle Zusatzdienstleistungen wie Beratung und Weiterbildung für alle Mitglieder des Praxisteams, Unterstützung bei der nachhaltigen Reduktion des Praxisaufwandes und Beratung bei Praxis- und Laborbedarf.
Die Unilabs-Strategie zielt darauf ab, ihre vielfältigen und regionalen Dienstleistungen über eine fundierte wissenschaftliche Struktur sicherzustellen. Zur Gewährleistung einer hochstehenden Qualität werden stets die notwendigen Investitionen für Ausrüstung und Ausbildung getätigt.

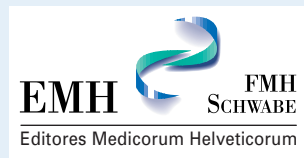

EMH Schweizerischer Ärzteverlag AG

Farnsburgerstrasse 8, 4132 Muttenz

Tel. 06146785 55, Fax 0614678556

verlag@emh.ch

www.emh.ch

\section{EMH, der Verlag der Ärztinnen und Ärzte}

Der Verlag EMH Schweizerischer Ärzteverlag AG wurde 1997 gegründet. EMH ist ein Gemeinschaftsunternehmen der Verbindung der Schweizer Ärztinnen und Ärzte FMH und der Schwabe AG, Basel, dem mit Gründung 1488 ältesten Druck- und Verlagshaus der Welt.

Hauptpublikationen von EMH sind die Zeitschriften «Schweizerische Ärztezeitung», das offizielle Publikationsorgan der FMH, «Swiss Medical Forum» mit praxisorientierten Fortbildungsbeiträgen, sowie «Swiss Medical Weekly», die Plattform für klinisch orientierte Wissenschaftler. Ebenfalls zu den Hauptpublikationen zählt «PrimaryCare», die offizielle «Schweizerische Zeitschrift für Hausarztmedizin».

Als erfolgreiches Online-Angebot ist unter anderem die Fortbildung des «Swiss Medical Forum» unter www.smf-cme.ch zu nennen. Steigende Zugriffszahlen und die Akkreditierung durch die Fachgesellschaften SGAM und SGIM als strukturierte und nachweisbare Fortbildung belegen diesen Erfolg.

Weitere medizinische Fachzeitschriften, ein ständig wachsendes Buchprogramm sowie viele Kooperationen und Dienstleistungen runden das umfangreiche Verlagsangebot ab.

\section{medica}

MEDIZINISCHE LABORATORIEN Dr. F. KAEPPELI AG

MEDIZINISCHE LABORATORIEN DR. F. KAEPPELI AG Eidgenössisch anerkannte Laboratorien

Wolfbachstrasse 17, 8024 Zürich

Tel. 04426999 99, Fax 0442699909

info@medica-labor.ch

www.medica-labor.ch

Der promovierte Mikrobiologe und Biochemiker Dr. F. Käppeli, Laborspezialist FAMH, übernahm 1976 das heute über 50-jährige Unternehmen und gründete als dessen Leiter und Inhaber die Einzelfirma medica. Der wichtigste unternehmerische Leitgedanke von 
Dr. F. Käppeli heisst kontinuierliche Innovation und Schaffung wegweisender Standards auf allen Gebieten der Labormedizin: Mikrobiologie inklusive Parasitologie, Serologie, Immunologie, klinische Chemie, Hämatologie, molekulare Diagnostik und Pathologie in Human- und Veterinärmedizin. So entstand ein Kompetenz-Zentrum für Labordiagnostik von gesamtschweizerisch grosser Bedeutung. Die modernst ausgebauten Laboratorien werden laufend erweitert und befinden sich im Herzen von Zürich. Über 200 Angestellte der Partnerlabors, begleitet von Spezialisten aus Medizin, Pharmakologie, Naturwissenschaften und Technik, garantieren für höchste Professionalität.$$
30 \text { mal }
$$

MCL Medizinische Laboratorien AG

Freiburgstrasse 634, 3172 Niederwangen

Tel. 03132878 78, Fax 0313287880

info@mcl.ch

www.mcl.ch

MCL ist ein Schweizer Unternehmen, das sich seit seiner Gründung 1981 in Familienbesitz befindet. Mit rund 150 Mitarbeitern bieten wir unsere Dienstleistungen in weiten Teilen der Schweiz an. Neben einem umfassenden Analysenspektrum bieten wir viele zusätzliche Dienstleistungen, dazu gehören moderne Kommunikationslösungen, vielfältige Fortbildungen oder die Beseitigung Ihrer biologischen Abfälle.

Wir sind immer in Ihrer Nähe - mit unseren Laboratorien und Blutentnahmezentren und auch als kompetenter, persönlicher Ansprechpartner für Ihre medizinischen Fragestellungen. Als unser Partner kontaktieren Sie unsere freundlichen Mitarbeiter über eine Gratis-Hotline.

Zusammen mit Aurigen, Dianalabs und Polyanalytic ist MCL Mitbegründerin eines schweizweit einzigartigen Labornetzwerks. Vom Austausch und der Nutzung der Synergien können Sie direkt profitieren: Neue Analysen und gesteigerte Analysenfrequenzen, neue Dienstleistungen und ein grösserer Expertenpool stehen zu Ihrer Verfügung.

\section{IVF HARTMANN AG}

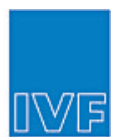

IVF HARTMANN AG

Victor-von-Bruns-Strasse 28, 8212 Neuhausen

Tel. 05267431 11, Fax 0526727441

info@ivf.hartmann.info

www.ivf.hartmann.info
Die IVF HARTMANN AG ist einer der führenden Anbieter für medizinische Verbrauchsgüter im Bereich Heilung, Pflege und Hygiene in der Schweiz. Ihre Lösungen helfen überall dort, wo Menschen geholfen wird. Zu ihren Kunden zählen somit Spitäler, Alters- und Pflegeheime, Spitex-Organisationen, niedergelassene Ärzte, Apotheken, Drogerien und der Lebensmitteleinzelhandel. Das breite Angebot der IVF HARTMANN AG umfasst über 2000 Produkte vom therapeutisch wirksamen Pflaster (z.B. Isola ${ }^{\circledR}$ Capsicum N) über funktionelle Verbände bis hin zu Produkten für die moderne Wundbehandlung (z.B. TenderWet ${ }^{\circledR}$ oder CompriGel $^{\circledR}$ ) und Erste Hilfe (z.B. DermaPlast ${ }^{\circledR}$ ). Die IVF HARTMANN GRUPPE ist eine 60-prozentige Tochtergesellschaft der PAUL HARTMANN AG mit Sitz in Heidenheim an der Brenz (D) und beschäftigt rund 350 Mitarbeiter. Neben ihrem Hauptsitz in Neuhausen am Rheinfall (SH) verfügt die IVF HARTMANN AG über weitere Produktionsstätten in Gommiswald (SG) und Netstal (GL).

\section{mepha}

Mepha Pharma AG

Dornacherstrasse 114, 4147 Aesch

Tel. 0617054343

www.mepha.ch

\section{Mepha - wir setzen Massstäbe}

Mepha, die führende Generika-Herstellerin der Schweiz, steht im 7. Jahrzehnt ihrer denkwürdigen Erfolgsgeschichte. Unseren Beitrag zu wirksamer Prophylaxe und Therapie sehen wir in der Entwicklung, Produktion und Vermarktung von günstigen, gut verträglichen und hochwertigen Generika. Wir entwickeln und produzieren in der Schweiz mit modernsten Hightechverfahren und nach höchstem Schweizer Qualitätsstandard. Unsere innovativen, kreativen Lösungen begeistern unsere Kunden immer wieder aufs Neue: zum Beispiel neuartige und verbesserte Anwendungsformen unserer Medikamente, die den Behandlungserfolg und das Wohlbefinden von Patientinnen und Patienten steigern. Alle unsere Leistungen gründen auf einer ganzheitlichen Sicht, welche die Interessen unserer Kunden, Mitarbeiter und Aktionäre, aber auch jene der übrigen Anspruchsgruppen in den Mittelpunkt stellt. Erstklassige Produkte, ein komplettes Package gefragter Dienstleistungen und offene Kommunikation sind weltweit Basis der Zufriedenheit unserer Kunden. 


\section{Salzmann ME DI C O}

Salzmann AG

Salzmann MEDICO

Rorschacher Strasse 304, 9016 St. Gallen

Tel. 07128212 12, Fax 0712821210

medico.sg@salzmann-group.ch

www.salzmann-group.ch

Salzmann MEDICO wurde 1980 durch Herrn Daniel Künzli, Präsident der Salzmann Group, gegründet. Die sehr kundenorientierte Handelsfirma vertreibt medizinische Verbrauchsgüter und Einwegprodukte. Die innovativen medizinischen Kompressionsstrümpfe der Marken VENOSAN ${ }^{\circledR}$ und VENOFIT ${ }^{\circledast}$ aus der Produktion von Salzmann MESH werden weltweit exportiert.

Produktesortiment: Produkte aus Produktion der Salzmann Abteilung MESH Marke VENOSAN ${ }^{\circledR}$; EXklusiv-Vertretungen unter Original-Markennamen; Private Label Produkte (SAMA ${ }^{\circledR}$, SAMA Orthopaedics $^{\circledR}, \quad$ Tale ${ }^{\circledR}$, Thermoban $\left.{ }^{\circledR}\right) ; \quad$ Wundkompressen; Wundtupfer; diverse Verbandsmaterialien; Heftpflaster / Wundschnellverbände; elastische Binden; medizinische Kompressionsstrümpfe / Stützstrümpfe; Körperbandagen, Orthesen, Schienen; Chirurgisches Nahtmaterial; Fixationsprodukte (Gips / synthetische Steifverbände); OP-Handschuhe; OP-Abdeckungen / OP-Bekleidung; OP-Sets steril; Produkte für die Sterilisation und Sterilisations-Kontrolle; Inkontinenzprodukte.

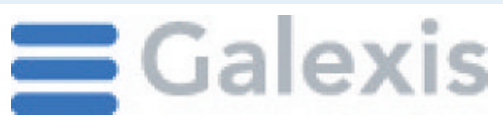

Galexis AG

Industriestrasse 2, Postfach, 4704 Niederbipp

Tel. 05885171 11, Fax 0588517114

info@galexis.com

www.galexis.com

Als Vollgrossist setzt Galexis AG Standards im Schweizer Gesundheitsmarkt. Wir beliefern unsere Kunden ganz nach dem Motto «Alles aus einer Hand» mit Pharma, Praxis- und Laborbedarf sowie Medizintechnik und erbringen darüber hinaus integrierte Dienstleistungen in der Gesundheitslogistik - schweizweit. Mit erprobten Lösungen fördert Galexis den Erfolg ihrer Kunden.

Möchten Sie ausserdem Ihre eigene Praxis praktisch, funktionell und ästhetisch einrichten? Genau hier kann Sie Galexis mit ihren Fachpartnern und einer langjährigen Erfahrung professionell beraten und unterstützen!

Überzeugen Sie sich - mit Galexis können Sie rechnen!

\section{MSD}

MSD Merck Sharp \& Dohme-Chibret AG

Schaffhauserstrasse 136, 8152 Opfikon-Glattbrugg Tel. 04482871 11, Fax 0448287210

www.msd.ch

www.univadis.ch

MSD ist die Schweizer Niederlassung von Merck \& Co., Inc. Whitehouse Station mit Hauptsitz in New Jersey, USA.

\section{DEM PATIENTEN VERPFLICHTET.}

Das Wohl des Patienten steht in unserer täglichen Arbeit an erster Stelle.

Als weltweit tätiger, forschender Arzneimittelhersteller entwickeln, produzieren und vertreiben wir innovative Medikamente und Impfstoffe. Wir tun dies seit mehr als 100 Jahren und heute in über 20 Therapiegebieten.

In unserer Verpflichtung dem Patienten gegenüber ermöglichen wir weltweit die Versorgung mit dringend benötigten Medikamenten und unterstützen nachhaltige Gesundheitsprogramme vor Ort.

\section{RÜECGE MEDICAL SYSTEMS}

Rüegge Medical Systems AG Seestrasse 86, Postfach 261, 8712 Stäfa Tel. 04347720 50, Fax 0434772060 info@medicalsystems.ch www.RadiologySystems.ch

\section{Unternehmensgeschichte}

Gründung am 18.7.1952 der Adolf Rüegge Ing., Baden. Damit ist die heutige Rüegge Medical Systems AG, Stäfa, die traditionsreichste, nicht zu einem internationalen Grosskonzern gehörende Radiologiefirma der Schweiz. Das Unternehmen verfügt über ein flächendeckendes Servicenetz mit dezentralen Servicestellen in allen 3 Landesteilen.

Heutige Aktivitäten der Rüegge Medical Systems Gruppe:

- Vertrieb, Installation und Wartung in der ganzen Schweiz von Digitalen Röntgensystemen, DR- und CR-Systemen weltweit führender Hersteller

- Vertrieb, Installation und Wartung in der ganzen Schweiz von DICOM-PACS / DICOM miniPACS integrierten IT-Lösungen für Arztpraxen und Kliniken. 